\title{
On existence of the resolvent and discreteness of the spectrum of a class of differential operators of hyperbolic type
}

\author{
M. B. Muratbekov ${ }^{1}$, M. M. Muratbekov ${ }^{2}$, A. M. Abylayeva ${ }^{3}$ \\ ${ }^{1}$ Taraz State Pedagogical Institute, Tole bi 62, Taraz, Kazakhstan, 080000 \\ ${ }^{2,3}$ L. N. Gumilyev Eurasian National University, Munaitpasov 5, Astana, Kazakhstan, 010008
}

\begin{abstract}
The existence and compactness of the resolvent are studied in this paper. One of the main results is the criterion of discreteness of the spectrum of a hyperbolic singular differential operator.
\end{abstract}

Keywords: spectrum; resolvent; singular differential operator; hyperbolic type.

MSC 2010: 47A10, 35L81.

Singular differential operators, for example operators defined in an unbounded domain, in general may have not only a discrete but also a continuous spectrum. Therefore in general an arbitrary function cannot be decomposed into a series of eigenfunctions. For this reason the most important problem in the study of the spectrum in dependence of the behavior of the coefficients in the case of an unbounded domain is the discreteness of the spectrum.

Spectral characteristics of singular elliptic differential operators are well-studied and the typical difficulties encountered in connection with bad behaving coefficients clarified. An extensive literature is devoted to their study and we mention [1-3].

Review of the literature shows that such questions as: 1) the existence and compactness of the resolvent, 2) the discreteness of the spectrum of hyperbolic differential operators defined in an unbounded domain are not well studied.

We consider in the space $L_{2}(\Omega)$ the differential operator of hyperbolic type

$$
A_{0} u=u_{x x}-u_{y y}+a(y) u_{x}+c(y) u
$$

with the domain $D\left(A_{0}\right)$ of infinitely differentiable functions satisfying the conditions $u(-\pi ; y)=u(\pi ; y), \quad u_{x}(-\pi ; y)=u_{x}(\pi ; y)$ and compactly supported with respect to the variable $y$, where

$$
\Omega=\{(x, y):-\pi<x<\pi,-\infty<y<\infty\} .
$$

Further, we assume that the coefficients $a(y), c(y)$ satisfy the conditions:

i) $|a(y)| \geq \delta_{0}>0, \quad c(y) \geq \delta>0$ are continuous functions in $R=(-\infty ; \infty)$.

It is easy to verify that the operator $A_{0}$ admits closure in the space $L_{2}(\Omega)$, which is denoted by $A$.

We note that the operator $A$ corresponds to the problem of propagation of the boundary regime (see [1], p. 106), i.e. the problem without initial conditions. Here the term $a u_{x}$ describes the friction force. The question of the existence of solutions of the problem without initial conditions, in general, depends on the behavior of the coefficients $a$ and $c$. For example, when $a=0$, the solution does not always exist.

The main results of this paper are the following theorems.

\footnotetext{
${ }^{1}$ Email: musahan_m@mail.ru

${ }^{2}$ Email: muratbekov_mm@enu.kz

${ }^{3}$ Email: abylayeva_b@mail.ru
} 
Theorem 1.1 Let the condition i) be fulfilled. Then the operator $A+\lambda I$ is continuously invertible for $\lambda \geq 0$.

Theorem 1.2 Let the condition i) be fulfilled. Then the resolvent of the operator $A$ is compact if and only if for any $w>0$

$$
\lim _{|y| \rightarrow \infty} \int_{y}^{y+w} c(t) d t=\infty .
$$

The last theorem shows that the condition $(*)$ is a necessary and sufficient condition for the discreteness of the spectrum of $A$.

The question of the existence of the resolvent and discrete spectrum in an unbounded domain with growing and oscillating coefficients was previously studied only in the case of elliptic and pseudodifferential operators [1-3].

Assume that the coefficients of the operator $A$, in addition to conditions $i$ ), satisfy the condition

$$
\text { ii) } \mu_{0}=\sup _{|y-t| \leq 1} \frac{c(y)}{c(t)}<\infty, \mu=\sup _{|y-t| \leq 1} \frac{a(y)}{a(t)}<\infty \text {. }
$$

Then, Theorem 1.2 easily implies the following theorem.

Theorem 1.3 Let the conditions i)-ii) be fulfilled. Then the resolvent of the operator $A$ is compact if and only if $\lim _{|y| \rightarrow \infty} c(y)=\infty$.

\section{Auxiliary lemmas and inequalities}

To prove the following statements below, we use computations and arguments that have been used in [5].

Lemma 2.1 Let the condition i) be fulfilled and $\lambda \geq 0$. Then the inequality

$$
\|(A+\lambda I) u\|_{2} \geq c\|u\|_{2},
$$

holds for all $u \in D(A)$, where $c=c\left(\delta, \delta_{0}\right)$ and $\|\cdot\|_{2}$ is the norm in $L_{2}(\Omega)$.

Proof. Let $u \in C_{0, \pi}^{\infty}(\bar{\Omega})$, where $C_{0, \pi}^{\infty}(\bar{\Omega})$ is a space of infinitely differentiable functions satisfying the conditions $u(-\pi ; y)=u(\pi ; y), u_{x}(-\pi ; y)=u_{x}(\pi ; y)$ and compactly supported with respect to the variable $y$. Integrating by parts the expressions $\langle(A+\lambda I) u, u\rangle$ and $\left\langle(A+\lambda I) u, u_{x}\right\rangle$ we obtain the following inequalities

$$
\begin{gathered}
\frac{1}{2 \varepsilon}\|(A+\lambda I) u\|_{2}^{2} \geq \int_{\Omega}\left[\left|u_{y}\right|^{2}+\left(\delta+\lambda-\frac{\varepsilon}{2}\right)|u|^{2}\right] d x d y-\int_{\Omega}\left|u_{x}\right|^{2} d x d y, \\
\|(A+\lambda I) u\|_{2}^{2} \geq \delta_{0}^{2}\left\|u_{x}\right\|_{2}^{2},
\end{gathered}
$$

where $\langle\cdot, \cdot\rangle$ is the scalar product in $L_{2}(\Omega)$.

Here we used the Cauchy inequality, with $\varepsilon=\frac{\delta}{2}>0$. From (2.2) and (2.3) the estimate $\|(A+\lambda I) u\|_{2}^{2} \geq c\|u\|_{2}^{2}$ follows. Since $A+\lambda I$ is the closed operator the last estimate holds for all $u \in D(A+\lambda I)$. 
Let $\Delta_{j}=(j-1, j+1)(j \in Z)$, and $\gamma$ be a constant such that $\gamma a(y)>0$. Denote by $l_{n, j, \gamma}+\lambda I$ the closure in $L_{2}\left(\Delta_{j}\right)$ of the differential expression

$$
\left(l_{n, j, \gamma}+\lambda I\right) u=-u^{\prime \prime}+\left[-n^{2}+i n(a(y)+\gamma)+c(y)+\lambda\right] u, \quad(n=0, \pm 1, \pm 2, \ldots)
$$

defined on the set $C_{0}^{2}\left(\bar{\Delta}_{j}\right)$ of twice continuously differentiable functions $u$ on $\bar{\Delta}_{j}$ which satisfy the conditions $u(j-1)=u(j+1)=0$.

Lemma 2.2 Let the condition i) be fulfilled and $\lambda \geq 0$. Then the inequalities

a)

$$
\begin{aligned}
& \left\|\left(l_{n, \gamma, j}+\lambda I\right) u\right\|_{L_{2}\left(\Delta_{j}\right)} \geq c_{1}\left(\left\|u^{\prime}\right\|_{2}+\|\sqrt{c(y)+\lambda} u\|_{L_{2}\left(\Delta_{j}\right)}+\right. \\
& \left.+\||n| \sqrt{(|a(y)|+|\gamma|)} u\|_{L_{2}\left(\Delta_{j}\right)}\right), n \neq 0, u \in D\left(l_{n, \gamma, j}+\lambda I\right) ;
\end{aligned}
$$

b) $\left\|\left(l_{n, j, \gamma}+\lambda I\right)^{-1}\right\|_{L_{2}\left(\Delta_{j}\right) \rightarrow L_{2}\left(\Delta_{j}\right)} \leq \frac{c_{0}}{(\delta+\lambda)^{1 / 2}}$;

c) $\left\|\frac{d}{d y}\left(l_{n, j, \gamma}+\lambda I\right)^{-1}\right\|_{L_{2}\left(\Delta_{j}\right) \rightarrow L_{2}\left(\Delta_{j}\right)} \leq \frac{c_{2}}{(\delta+\lambda)^{1 / 4}}$

hold, where $c_{0}=c_{0}(\delta), c_{1}=c_{1}(\delta), c_{2}=c_{2}(\delta)$.

Proof. Let $u \in C_{0}^{2}\left(\Delta_{j}\right)$. We have

$$
\left|\left\langle\left(l_{n, j, \gamma}+\lambda I\right) u, u\right\rangle\right| \geq\left.\left|\left\|u^{\prime}\right\|_{L_{2}\left(\Delta_{j}\right)}^{2}+\int_{\Delta_{j}}(c(y)+\lambda)\right| u\right|^{2} d y|-| \int_{\Delta_{j}} n^{2}|u|^{2} d y \mid .
$$

Hence

$$
\left\|\left(l_{n, j, \gamma}+\lambda I\right) u\right\|_{L_{2}\left(\Delta_{j}\right)} \cdot\|u\|_{L_{2}\left(\Delta_{j}\right)} \geq \int_{\Delta_{j}}\left|u^{\prime}\right|^{2} d y-\int_{\Delta_{j}}|n|^{2}|u|^{2} d y
$$

and

$$
\frac{1}{\delta}\left\|\left(l_{n, j, \gamma}+\lambda I\right) u\right\|_{L_{2}\left(\Delta_{j}\right)}^{2} \geq \frac{1}{2} \int_{\Delta_{j}}(c(y)+\lambda)|u|^{2} d y-\int_{\Delta_{j}}|n|^{2}|u|^{2} d y .
$$

Here we again used the Cauchy inequality with $\varepsilon>0$, where $\varepsilon=\frac{\delta}{2}>0$.

On the other hand, by transforming the expression $\left\langle\left(l_{n, j, \gamma}+\lambda I\right) u, u\right\rangle_{\Delta_{j}}$ and $\left\langle\left(l_{n, j, \gamma}+\right.\right.$ $\lambda I) u,-i n u\rangle_{\Delta_{j}}, u \in C_{0}^{2}\left(\bar{\Delta}_{j}\right)$, we have

$$
c\left(\delta_{0}\right)\left\|\left(l_{n, j, \gamma}+\lambda I\right) u\right\|_{L_{2}\left(\Delta_{j}\right)} \geq\|\sqrt{|n|(a(y)+|\gamma|)} u\|_{L_{2}\left(\Delta_{j}\right)}
$$

and

$$
\left\|\left(l_{n, j, \gamma}+\lambda I\right) u\right\|_{L_{2}\left(\Delta_{j}\right)}^{2} \geq\left(\delta_{0}+|\gamma|\right)^{2} \cdot|n|^{2}\|u\|_{L_{2}\left(\Delta_{j}\right)}^{2} .
$$

Combining (2.6) with (2.8), and choosing $\gamma$ so that $\left(\delta_{0}+|\gamma|\right)^{2}-1 \geq 0$, we obtain

$$
c_{0}(\delta)\left\|\left(l_{n, j, \gamma}+\lambda I\right) u\right\|_{L_{2}\left(\Delta_{j}\right)} \geq\|\sqrt{c(y)+\lambda} u\|_{L_{2}\left(\Delta_{j}\right)},
$$

where $c_{0}(\delta)=2\left(\frac{1}{\delta}+1\right)$. Hence by the conditions $\left.i\right)$ we conclude

$$
\frac{c_{0}(\delta)}{\sqrt{\delta+\lambda}}\left\|\left(l_{n, j, \gamma}+\lambda I\right) u\right\|_{L_{2}\left(\Delta_{j}\right)} \geq\|u\|_{L_{2}\left(\Delta_{j}\right)} .
$$

From inequalities (2.5), (2.8) and (2.10) we obtain the estimate

$$
\frac{c_{0}(\delta)+1}{\sqrt{\delta+\lambda}}\left\|\left(l_{n, j, \gamma}+\lambda I\right) u\right\|_{L_{2}\left(\Delta_{j}\right)}^{2} \geq \int_{\Delta_{j}}\left|u^{\prime}\right|^{2} d y+|n|^{2} \int_{\Delta_{j}}\left(\frac{\left(\delta_{0}+|\gamma|\right)^{2}}{\sqrt{\delta+\lambda}}-1\right)|u|^{2} d y
$$


The estimate $a$ ) follows from inequalities (2.7), (2.9) and (2.11) by choosing $\gamma$ so that $\frac{\left(\delta_{0}+|\gamma|\right)^{2}}{\sqrt{\delta+\lambda}}-1 \geq 0$. The estimate (2.10) implies $b$ ). From the inequality (2.11) it follows the estimate

$$
\frac{c_{2}(\delta)}{\sqrt{\delta+\lambda}}\left\|\left(l_{n, j, \gamma}+\lambda I\right) u\right\|_{L_{2}\left(\Delta_{j}\right)}^{2} \geq\left\|u^{\prime}\right\|_{L_{2}\left(\Delta_{j}\right)}^{2}, \quad c_{2}(\delta)=c_{0}(\delta)+1 .
$$

This implies the estimate $c$ ). Lemma 2.2 is completely proved.

Lemma 2.3 The operator $l_{n, j, \gamma}+\lambda I$ is invertible for $\lambda \geq 0$ and the inverse operator $\left(l_{n, j, \gamma}+\lambda I\right)^{-1}$ is defined in all $L_{2}\left(\Delta_{j}\right), j \in Z$.

Proof. By estimate $b)$ in Lemma 2.2 it is enough to prove that $R\left(l_{n, \gamma, j}+\lambda I\right)=L_{2}\left(\Delta_{j}\right)$, where $R\left(l_{n, \gamma, j}+\lambda I\right)$ is the range of the operator $l_{n, \gamma, j}+\lambda I$. Assume the contrary. Then there exists an element $v \in L_{2}\left(\Delta_{j}\right), v \neq 0$, which satisfies the equation

$$
\left(l_{n, \gamma, j}+\lambda I\right)^{*} v=-v^{\prime \prime}+\left[-n^{2}-i n(a(y)+\gamma)+c(y)+\lambda\right] v=0
$$

in the sense of the theory of distributions. This implies that $v^{\prime \prime} \in L_{2}\left(\Delta_{j}\right)$. Integrating by parts the expression $\left\langle\left(l_{n, j, \gamma}+\lambda I\right) u, v\right\rangle_{\Delta_{j}}$ we have $u^{\prime}(j+1) \bar{v}(j+1)-u^{\prime}(j-1) \bar{v}(j-1)=0$, where $u$ is an arbitrary function from $C_{0}^{\infty}\left(\Delta_{j}\right)$. Therefore $v(j+1)=v(j-1)=0$, and using these conditions we can derive the inequality

$$
\left\|\left(l_{n, \gamma, j}+\lambda I\right)^{*} v\right\|_{L_{2}\left(\Delta_{j}\right)}^{2} \geq c\|v\|_{L_{2}\left(\Delta_{j}\right)}^{2},
$$

similarly to (2.10). This implies that $v=0$. Lemma is proved.

By $l_{n, \gamma}+\lambda I(n=0, \pm 1, \pm 2, \ldots)$ we denote the closure in $L_{2}(R)$ of the differential expression $\left(l_{n, \gamma}+\lambda I\right) u=-u^{\prime \prime}+\left(-n^{2}+i n(a(y)+\gamma)+c(y)+\lambda\right) u$, defined on the set $C_{0}^{\infty}(R)$ of infinitely differentiable functions with compact support.

Lemma 2.4 Let $\lambda \geq 0$ and condition $i)$ hold. Then for any $u \in D\left(l_{n, \gamma}+\lambda I\right)$ the estimates

$$
\begin{aligned}
& \left\|\left(l_{0, \gamma}+\lambda I\right) u\right\|_{L_{2}(R)} \geq \sqrt{\delta+\lambda}\|u\|_{L_{2}(R)}, \\
& \left\|\left(l_{n, \gamma}+\lambda I\right) u\right\|_{L_{2}(R)} \geq|n|\left(\delta_{0}+|\gamma|\right)\|u\|_{L_{2}(R)}
\end{aligned}
$$

hold for $n \neq 0$.

Lemma 2.4 is proved by transforming the expression $\left\langle\left(l_{n, \gamma}+\lambda I\right) u,-i n u\right\rangle$, where $u \in$ $C_{0}^{\infty}(R)$.

Let $\left\{\varphi_{j}(y)\right\}_{j=-\infty}^{+\infty} \subset C_{0}^{\infty}(R)$ be a sequence of functions satisfying the conditions $\varphi_{j} \geq 0$, $\operatorname{supp} \varphi_{j} \subseteq \Delta_{j}(j \in Z), \sum_{j=-\infty}^{+\infty} \varphi_{j}^{2}(y)=1$. Assume

$$
\begin{gathered}
K_{\lambda, \gamma} f=\sum_{j=-\infty}^{+\infty} \varphi_{j}\left(l_{n, j, \gamma}+\lambda I\right)^{-1} \varphi_{j} f, \\
B_{\lambda, \gamma} f=\sum_{j=-\infty}^{+\infty} \varphi_{j}^{\prime \prime}\left(l_{n, j, \gamma}+\lambda I\right)^{-1} \varphi_{j} f+2 \sum_{j=-\infty}^{+\infty} \varphi_{j}^{\prime} \frac{d}{d y}\left(l_{n, j, \gamma}+\lambda I\right)^{-1} \varphi_{j} f, f \in C_{0}^{\infty}(R), \\
\lambda \geq 0 .
\end{gathered}
$$

Obviously

$$
\left(l_{n, \gamma}+\lambda I\right) K_{\lambda, \gamma} f=f-B_{\lambda, \gamma} f .
$$

Lemma 2.5 Let the condition i) be fulfilled. Then there exists a number $\lambda_{0}>0$ such that $\left\|B_{\lambda, \gamma}\right\|_{L_{2}(R) \rightarrow L_{2}(R)}<1$ for all $\lambda \geq \lambda_{0}$. 
Proof. Let $f \in C_{0}^{\infty}(R)$. Since only the functions $\varphi_{j-1}, \varphi_{j}, \varphi_{j+1}$ can be nonzero on $\bar{\Delta}_{j}(j \in Z)$ we have

$$
\begin{aligned}
& \left\|B_{\lambda, \gamma} f\right\|_{L_{2}(R)}^{2}= \\
& =\int_{-\infty}^{\infty}\left|\sum_{j=-\infty}^{\infty} \varphi_{j}^{\prime \prime}\left(l_{n, j, \gamma}+\lambda I\right)^{-1} \varphi_{j} f+2 \sum_{j=-\infty}^{\infty} \varphi_{j}^{\prime} \frac{d}{d y}\left(l_{n, j, \gamma}+\lambda I\right)^{-1} \varphi_{j} f\right|^{2} d y \leq \\
& \leq \sum_{j=-\infty}^{+\infty} \int_{-\infty}^{+\infty}\left|\sum_{k=j-1}^{j+1}\left[\varphi_{k}^{\prime \prime}\left(l_{n, k, \gamma}+\lambda I\right)^{-1} \varphi_{k} f+2 \varphi_{k}^{\prime} \frac{d}{d y}\left(l_{n, k, \gamma}+\lambda I\right)^{-1} \varphi_{k} f\right]\right|^{2} d y .
\end{aligned}
$$

Hence using the obvious inequality $(a+b+c)^{2} \leq 3\left(a^{2}+b^{2}+c^{2}\right)$ and estimates $\left.b\right), c$ ) in Lemma 2.2, we obtain

$$
\left\|B_{\lambda, \gamma} f\right\|_{L_{2}(R)}^{2} \leq c\left(\frac{c_{0}}{(\delta+\lambda)^{1 / 2}}+\frac{c_{2}}{(\delta+\lambda)^{1 / 4}}\right)\|f\|_{L_{2}(R)}^{2},
$$

where $c=24 \max \left\{\left|\varphi_{j}^{\prime \prime}\right|,\left|\varphi_{j}^{\prime}\right|\right\}$, and the constants $c_{0}, c_{2}$ are from Lemma 2.2. Hence, it is easy to choose a number $\lambda_{0}>0$ such that $\left\|B_{\lambda, \gamma}\right\|_{L_{2}(R) \rightarrow L_{2}(R)}<1$ for $\lambda \geq \lambda_{0}$. This completes the proof.

Lemma 2.6 Let the condition i) be satisfied. Then the operator $l_{n, \gamma}+\lambda I$ is continuously invertible for $\lambda \geq \lambda_{0}>0$, and for the inverse operator $\left(l_{n, \gamma}+\lambda I\right)^{-1}$ the equality

$$
\left(l_{n, \gamma}+\lambda I\right)^{-1}=K_{\lambda, \gamma}\left(I-B_{\lambda, \gamma}\right)^{-1}
$$

holds.

Lemma 2.6 follows from (2.14) and from Lemmas 2.5 and 2.4.

Lemma 2.7 Let the condition i) be satisfied and $\rho(y)$ be a continuous function defined on $R$. Then for $\alpha=0,1$ and $\lambda \geq \lambda_{0}$ the estimate

$$
\begin{aligned}
& \left\|\rho(y)|n|^{\alpha}\left(l_{n, \gamma}+\lambda I\right)^{-1}\right\|_{L_{2}(R) \rightarrow L_{2}(R)}^{2} \leq \\
\leq & c_{4}(\lambda) \sup _{j \in Z}\left\|\rho(y)|n|^{\alpha} \varphi_{j}\left(l_{n, j, \gamma}+\lambda I\right)^{-1}\right\|_{L_{2}\left(\Delta_{j}\right) \rightarrow L_{2}\left(\Delta_{j}\right)}^{2}
\end{aligned}
$$

holds.

Proof. Let $f \in C_{0}^{\infty}(R)$. From representation (2.15) and by the properties of the functions $\left\{\varphi_{j}\right\}(j \in Z)$, we have

$$
\begin{aligned}
& \left\|\rho(y)|n|^{\alpha}\left(l_{n, \gamma}+\lambda I\right)^{-1} f\right\|_{L_{2}(R)}^{2} \leq \\
\leq & \sum_{j=-\infty}^{+\infty} \int_{-\infty}^{+\infty}\left|\sum_{k=j-1}^{j+1}\left[\rho(y)|n|^{\alpha} \varphi_{k}\left(l_{n, k, \gamma}+\lambda I\right)^{-1} \varphi_{k}\left(I-B_{\lambda, \gamma}\right)^{-1} f\right]\right|^{2} d y .
\end{aligned}
$$

Hence, using again the inequality $\left(a_{0}+b_{0}+c_{0}\right)^{2} \leq 3\left(a_{0}^{2}+b_{0}^{2}+c_{0}^{2}\right)$ and by Lemma 2.5 , we obtain the estimate (2.16). Lemma 2.7 is proved.

The result below follows from Lemma 2.2 and the estimate (2.16).

Lemma 2.8 Let the condition i) be satisfied and $\lambda \geq \lambda_{0}$. Then

$$
\begin{aligned}
& \text { a) }\left\|\sqrt{c(y)+\lambda}\left(l_{n, \gamma}+\lambda I\right)^{-1}\right\|_{L_{2}(R) \rightarrow L_{2}(R)}<\infty(n=0, \pm 1, \pm 2, \ldots) ; \\
& \text { b) }\left\|i n\left(l_{n, \gamma}+\lambda I\right)^{-1}\right\|_{L_{2}(R) \rightarrow L_{2}(R)}<\infty(n \neq 0) ; \\
& \text { c) }\left\|\frac{d}{d y}\left(l_{n, \gamma}+\lambda I\right)^{-1}\right\|_{L_{2}(R) \rightarrow L_{2}(R)}<\infty(n=0, \pm 1, \pm 2, \ldots) .
\end{aligned}
$$


Consider the equation

$$
\left(l_{n}+\lambda I\right) u \equiv-u^{\prime \prime}+\left(-n^{2}+i n a(y)+c(y)+\lambda\right) u=f,
$$

where $f \in L_{2}(R)$.

The function $u \in L_{2}(R)$ is called a solution of the equation (2.17) if there exists a sequence $\left\{u_{n}\right\}_{n=1}^{\infty} \subset C_{0}^{\infty}(R)$ such that $\left\|u_{n}-u\right\|_{L_{2}(R)} \rightarrow 0,\left\|\left(l_{n}+\lambda I\right) u_{n}-f\right\|_{L_{2}(R)} \rightarrow 0$ as $n \rightarrow \infty$.

Lemma 2.9 The operator $l_{n}+\lambda I(n=0, \pm 1, \pm 2, \ldots)$ is boundedly invertible for $\lambda \geq \lambda_{0}$, and for the inverse operator $\left(l_{n}+\lambda I\right)^{-1}$ the equality

$$
\left(l_{n}+\lambda I\right)^{-1} f=\left(l_{n, \gamma}+\lambda I\right)^{-1}\left(I-A_{\lambda, \gamma}\right)^{-1} f, f \in L_{2}(R)
$$

holds, where $\left\|A_{\lambda, \gamma}\right\|_{L_{2}(R) \rightarrow L_{2}(R)}<1$.

Proof. Let $n \neq 0$. We rewrite the equation $\left(l_{n}+\lambda I\right) u=f \in L_{2}(R)$ in the form $v-A_{\lambda, \gamma} v=f$, where $v=\left(l_{n, \gamma}+\lambda I\right) u, A_{\lambda, \gamma}=i n \gamma\left(l_{n, \gamma}+\lambda I\right)^{-1}$. From Lemma 2.4 it follows that $\left\|A_{\lambda, \gamma} v\right\|_{L_{2}(R) \rightarrow L_{2}(R)} \leq \frac{|n| \cdot|\gamma|}{|n|\left(\delta_{0}+|\gamma|\right)}\|v\|_{L_{2}(R)}$ or $\left\|A_{\lambda, \gamma}\right\|_{L_{2}(R) \rightarrow L_{2}(R)}<1$. Hence

$$
u=\left(l_{n}+\lambda I\right)^{-1} v=\left(l_{n, \gamma}+\lambda I\right)^{-1}\left(I-A_{\lambda, \gamma}\right)^{-1} f .
$$

The operator $l_{0}+\lambda I$ be a self-adjoint operator for $n=0[6$, p. 208] and the estimate $\left\|\left(l_{0}+\lambda I\right) u\right\|_{L_{2}(R)} \geq(\delta+\lambda)\|u\|_{L_{2}(R)}$ holds for any $u \in D\left(l_{0}+\lambda I\right)$. These imply that the operator $l_{0}+\lambda I$ is boundedly invertible in all $L_{2}(R)$. Lemma 2.9 is proved.

Lemma 2.8 and the equality (2.18) imply the following lemma.

Lemma 2.10 If $\lambda \geq \lambda_{0}$, then the estimates
a) $\left\|\sqrt{c(y)+\lambda}\left(l_{n}+\lambda I\right)^{-1}\right\|_{L_{2}(R) \rightarrow L_{2}(R)}<\infty(n=0, \pm 1, \pm 2, \ldots)$;
b) $\|$ in $\left(l_{n}+\lambda I\right)^{-1} \|_{L_{2}(R) \rightarrow L_{2}(R)}<\infty(n \neq 0)$;
c) $\left\|\frac{d}{d y}\left(l_{n}+\lambda I\right)^{-1}\right\|_{L_{2}(R) \rightarrow L_{2}(R)}<\infty(n=0, \pm 1, \pm 2, \ldots)$ hold.

We will use also the following well-known lemma [7, p. 350].

Lemma 2.11 Let the operator $A+\lambda_{0} I \quad\left(\lambda_{0}>0\right)$ be boundedly invertible in $L_{2}(R)$ and the estimate $\|(A+\lambda I) u\|_{L_{2}(R)} \geq c\|u\|_{L_{2}(R)}, u \in D(A+\lambda I)$ hold for $\lambda \in\left(0, \lambda_{0}\right]$. Then the operator $A: L_{2}(R) \rightarrow L_{2}(R)$ is boundedly invertible also.

Lemma 2.12 Let the condition i) be fulfilled and $\lambda>0$. Then the inequality

$$
\left\|\left(l_{n}+\lambda I\right)^{-1}\right\|_{2 \rightarrow 2} \leq \frac{1}{|n| \cdot \delta_{0}}
$$

holds for all $n(n=0, \pm 1, \pm 2, \ldots)$.

Proof. Let $u \in C_{0}^{\infty}(R)$. Integrating the expression $\left\langle\left(l_{n}+\lambda I\right) u, u\right\rangle$ by parts, we obtain

$$
\left|\left\langle\left(l_{n}+\lambda I\right) u, u\right\rangle\right|=\left.\left|\int_{-\infty}^{\infty}\left[\left|u^{\prime}\right|^{2}+\left(-n^{2}+c(y)\right)|u|^{2}\right] d y+\int_{-\infty}^{\infty} \operatorname{ina}(y)\right| u\right|^{2} d y \mid .
$$

Hence, taking $i$ ) into account and using the property of complex numbers, we have

$$
\left\|\left(l_{n}+\lambda I\right) u\right\|_{2} \geq|n| \cdot \delta_{0}\|u\|_{2} .
$$

It is taken into account that the sign of $a(y)$ does not change. The inequality $(2.20)$ implies the proof of Lemma 2.12. 
Lemma 2.13 Let the condition i) be fulfilled and $\lambda>0$. Then the operator $\left(l_{n}+\lambda I\right)^{-1}$ is completely continuous for all $n(n=0, \pm 1, \pm 2, \ldots)$ if and only if for any $\omega>0$

$$
\lim _{|y| \rightarrow \infty} \int_{y}^{y+\omega} c(t) d t=\infty
$$

Proof. From Lemma 2.12 it follows that it is sufficient to prove Lemma 2.13 for any finite $n \neq 0$.

Let $n=0$. Then the operator $l_{n}+\lambda I$ is an operator of Sturm-Liouville type with potential $c(y)$, i.e.

$$
\left(l_{0}+\lambda I\right) u=-u^{\prime \prime}+c(y) u, u \in D\left(l_{0}\right) .
$$

In this case, reproducing all the computations and arguments used in [1,2], we obtain the proof of Lemma 2.13, i.e. the equality $(*)$ is a necessary and sufficient condition for the compactness of the resolvent of the operator $l_{0}+\lambda I$.

Consider the case $n \neq 0$. Let

$$
\lim _{|y| \rightarrow \infty} \int_{y}^{y+w}(|n a(t)|+c(t)) d t=\infty .
$$

Necessity. Let the condition (2.21) be not satisfied. Then there exists a sequence of intervals $Q_{d}\left(y_{j}\right) \subset R$ such that

$$
\sup _{\{j\}} \int_{Q_{d}\left(y_{j}\right)}(|n a(t)|+c(t)) d t<\infty,
$$

for every finite $n$ where $d>0$, i.e. when the intervals $Q_{d}\left(y_{j}\right)$, preserving the length, converge to infinity. Let $w(x) \in C_{0}^{\infty}\left(Q_{d}(0)\right)$ and consider the set of functions such that $u_{j}(y)=w\left(y-y_{j}\right)$. It is easy to verify for every finite $n$ the following inequality

$$
\begin{aligned}
& \left\|-u_{j}^{\prime \prime}+\left(-n^{2}+i n a(y)+c(y)+\lambda\right) u_{j}(y)\right\|_{2}^{2}= \\
= & \int_{-\infty}^{\infty}\left|-u_{j}^{\prime \prime}(y)+\left(-n^{2}+i n a(y)+c(y)+\lambda\right) u_{j}(y)\right|^{2} d y \leq \\
\leq & 8 \int_{Q_{d}\left(y_{j}\right)}\left[\left|-u_{j}^{\prime \prime}(y)\right|^{2}+\left(n^{4}+n^{2} a^{2}(y)+c^{2}(y)+\lambda^{2}\right)\left|u_{j}(y)\right|^{2}\right] d y .
\end{aligned}
$$

From (2.23), taking the inequality (2.22) and the property of the function $u_{j}(y)=$ $\omega\left(y-y_{j}\right)$ into account, we obtain

$$
\left\|-u_{j}^{\prime \prime}+\left(-n^{2}+i n a(y)+c(y)+\lambda\right) u_{j}(y)\right\|_{2}^{2}<c<\infty,
$$

for every finite $n \neq 0$, where $c>0$ is independent of $j$.

We assume

$$
F_{j}(y)=-u_{j}^{\prime \prime}(y)+\left(-n^{2}+i n a(y)+c(y)+\lambda\right) u_{j}(y), \quad \operatorname{supp} F_{j}(y) \subseteq Q_{d}\left(y_{j}\right) .
$$

Now we show that $F_{j}(y)$ weakly converges to zero in $L_{2}(R)$.

$$
\begin{gathered}
\quad\left\langle F_{j}(y), v(y)\right\rangle=\left|\int_{-\infty}^{\infty} F_{j}(y) v(y) d y\right| \leq \\
\leq\left(\int_{Q_{d}\left(y_{j}\right)} F_{j}^{2}(y) d y\right)^{\frac{1}{2}}\left(\int_{Q_{d}\left(y_{j}\right)} v^{2}(y) d y\right)^{\frac{1}{2}}
\end{gathered}
$$


Since $v \in L_{2}(R)$, it is obvious that $\int_{Q_{d}\left(y_{j}\right)} v^{2}(y) d y \rightarrow 0$ as $j \rightarrow \infty$. Taking it into account, from $(2.24)$ we find that the sequence $\left\{F_{j}\right\}$ converges weakly to zero.

It is immediately clear that

$$
\left\|u_{j}(y)\right\|_{2}=\alpha>0 \text {. }
$$

Since, if the operator $\left(l_{n}+\lambda I\right)^{-1}$ is compact and then $\left\{u_{j}(y)\right\}$ should converge to zero in the norm $L_{2}(R)$. But this is impossible by (2.25), i.e. we have a contradiction.

Thus, we have proved that in case $n \neq 0$ the condition (2.21) is a necessary condition for the compactness of the resolvent $l_{n}+\lambda I$. From $(*)$ and $(2.21)$ it follows that the equality $(*)$ is a necessary condition for the compactness of the resolvent of the operator $l_{n}+\lambda I$ for all $n(n=0, \pm 1, \pm 2, \ldots)$.

Sufficiency. From Lemma 2.10 it follows that $R\left(l_{n}+\lambda I\right)^{-1} \subset L_{2}^{1}(R, c(y))$, where $R\left(l_{n}+\lambda I\right)^{-1}$ is the range of the operator $\left(l_{n}+\lambda I\right)^{-1}$, and $L_{2, c(y)}^{1}$ is the space obtained by completing $C_{0}^{\infty}(R)$ with respect to the norm

$$
\left\|u: L_{2, c(y)}^{1}\right\|=\left(\int_{-\infty}^{+\infty}\left(\left|u^{\prime}\right|^{2}+(c(y)+\lambda)|u|^{2}\right) d y\right)^{\frac{1}{2}} .
$$

To complete the proof it remains to show that the embedding operator of the space $L_{2, c(y)}^{1}$ in $L_{2}(R)$ is compact. The answer to this question follows from the results of [2]. In that paper it is shown that any bounded set of the space $L_{2, c(y)}^{1}$ is compact in $L_{2}(R)$ if and only if

$$
\lim _{|y| \rightarrow \infty} c^{*}(y)=\infty
$$

where the function $c^{*}(y)$ is a special averaging of the function $c(y)$ [2]. Further, in [8, p. 58] it is proved, that the conditions $(*)$ and (2.26) are equivalent. Sufficiency of Lemma 2.13 is proved.

\section{Proofs of Theorems 1.1-1.3}

From Lemma 2.9 we obtain that

$$
u_{k}(x, y)=\sum_{n=-k}^{k}\left(l_{n}+\lambda I\right)^{-1} f_{n}(y) e^{i n x}
$$

is a solution of the problem

$$
\begin{gathered}
(A+\lambda I) u_{k}(x, y)=f_{k}(x, y) \\
u_{k}(-\pi, y)=u_{k}(\pi, y), \quad u_{k x}(-\pi, y)=u_{k x}(\pi, y),
\end{gathered}
$$

where $f_{k}(x, y) \stackrel{L_{2}}{\longrightarrow} f(x, y), \quad f_{k}(x, y)=\sum_{n=-k}^{k} f_{n}(y) e^{i n x}, \quad\left(l_{n}+\lambda I\right)^{-1}$ is the inverse operator to the operator $\left(l_{n}+\lambda I\right)$.

By virtue of (2.1) we have

$$
\left\|u_{k}(x, y)\right\|_{2} \leq c\left\|f_{k}(x, y)\right\|_{2},
$$

where $c>0$ is a constant independent of $k$. 
Since $f_{k} \stackrel{L_{2}}{\longrightarrow} f$, then from $(3.2)$ we find

$$
\left\|u_{k}-u_{m}\right\|_{2} \leq c\left\|f_{k}-f_{m}\right\|_{2} \rightarrow 0 \text { as } k, m \rightarrow \infty .
$$

Hence, by virtue of the completeness of the space $L_{2}(\Omega)$, it follows that there exists a unique function $u \in L_{2}(\Omega)$ such that

$$
u_{k} \rightarrow u \text { as } k \rightarrow \infty
$$

(3.3) implies that for any $f \in L_{2}(\Omega)$

$$
u(x, y)=(A+\lambda I)^{-1} f(x, y)=\sum_{n=-\infty}^{\infty}\left(l_{n}+\lambda I\right)^{-1} f_{n}(y) e^{i n x}
$$

is a strong solution of the problem

$$
\begin{gathered}
(A+\lambda I) u=f \\
u(-\pi, y)=u(\pi, y), u_{x}(-\pi, y)=u_{x}(\pi, y)
\end{gathered}
$$

Let us recall the definition of strong solutions.

The function $u \in L_{2}(\Omega)$ is called a strong solution of (3.5)-(3.6), if there exists a sequence $\left\{u_{k}\right\}_{k=1}^{\infty} \subset D\left(L_{0}\right)$ such that $\left\|u_{k}-u\right\|_{2} \rightarrow 0,\left\|(A+\lambda I) u_{k}-f\right\|_{2} \rightarrow 0$ as $k \rightarrow \infty$.

Now, it is easy to see that (3.4) is the inverse operator to the closed operator $A+\lambda I$. Lemma 2.1 implies that the last statement holds for all $\lambda \geq 0$. Theorem 1.1 is proved.

Proof of Theorem 1.2. Using Lemma 2.12 it is easy to see that

$$
\lim _{|n| \rightarrow \infty}\left\|\left(l_{n}+\lambda I\right)^{-1}\right\|_{2 \rightarrow 2}=0
$$

Therefore, and using the $\varepsilon$-net, from (3.4) we have that the operator $(A+\lambda I)^{-1}$ is compact if and only if $\left(l_{n}+\lambda I\right)^{-1}$ is continuous. Now, the proof of the theorem follows from Lemma 2.13.

Proof of Theorem 1.2. Without loss of generality we assume $0<w \leq 1$, then by the condition $i i$ ) we have

$$
\mu_{0}^{-1} \cdot w \cdot c(y) \leq \int_{y}^{y+w} c(t) d t \leq \mu_{0} \cdot w \cdot c(y) .
$$

The proof of Theorem 1.3 follows from this inequality and Theorem 1.2.

\section{References}

[1] A. M. Molchanov. On conditions of the spectrum discreteness of self-adjoint secondorder differential equations. Trudy Mosc. Mat. Obshestva, 2 (1953) - P. 169-200 (in Russian).

[2] M. Otelbaev. Embedding theorems for spaces with a weight and their application to the study of the spectrum of the Schrodinger operator. Trudy Mat. Inst. Steklov, 150 (1979) - P. 256-305 (in Russian).

[3] K. Kh. Boimatov. Separation theorems, weighted spaces and their applications. Proc. Steklov Inst. Math. 170 (1987) - P. 39-81. 
[4] A. N. Tikhonov and A. A. Samarskiy. Equations of Mathematical Physics. Macmillan, State New York, 1963.

[5] M. Muratbekov, K. Ospanov, S. Igisinov. Solvability of a class of mixed type second order equations and nonlocal estimates. Applied Mathematics Letters. 25 (2012). P. 1661-1665.

[6] M. Reed, B. Simon. Methods of Modern Mathematical Physics. 1: Functional analysis, Academic Press, San Diego, 1980.

[7] N. I. Akhieser, I. M. Glasman. Theory of linear operators in Hilbert space. Originally Published: New York: F. Ungar Pub. Co., 1961-1963.

[8] M. Otelbaev. Estimates of the Spectrum of the Sturm-Liouville Operator. Gylym, Almaty, 1990 (in Russian).

(Received April 3, 2013) 